\title{
Gas Density Balance Design Considerations
}

\author{
E. C. Creitz \\ Institute for Applied Technology, National Bureau of Standards, Washington, D.C. 20234
}

(May 2, 1968)

\begin{abstract}
The Nerheim gas density balance operates by measuring the flow produced by differences in density between a sample and a reference gas in the earth's gravitational field. It has been considered theoretically in order to outline the characteristics of the gas flow system, those of the flow measuring anemometers, and the effects of detector volume on peak separation and sensitivity. It is shown that both design parameters and operating conditions affect the performance of the device, and that properly considered compromises can improve its characteristics.
\end{abstract}

Key Words: Chromatographic detector; density detector; gas density; gas detector.

\section{Introduction}

The Nerheim version [1] ${ }^{1}$ of the Martin gas density balance $[2,3]$ presents a number of advantages over other detectors for gas chromatography, not the least of which are its ease of calibration, and its adaptability to the measurement of corrosive column effluents. Ease of calibration is attributable to the "absolute" [4] nature of its output, being directly related to density difference between reference gas and column effluent. Corrosion resistance is achieved by handling the corrosive gases in ducts of resistant material so that the sample is not lost, while maintaining the sensing elements in an inert atmosphere, so that they, also, are not destroyed.

Nerheim [1] presented an analysis of the energy balance in the pneumatic parts of the detector. However, the information given was not of a type readily adaptable to instrument design. The study presented here was undertaken in an attempt to separate design parameters of a fixed nature from those which may be varied, and to define more clearly the variables affecting the overall response of the system to changes in density of the column effluent.

\section{Pneumatic System}

A schematic representation of the Nerheim gas density balance is shown in figure 1 , the lines representing ducts through which gases flow. The principle of operation is remarkably simple. Since its operation depends on gravitational effects, the duct system of figure 1 is arranged so that duct $\mathrm{BD}$, and those shown parallel to it, must be vertical. Reference gas enters

${ }^{1}$ Figures in brackets indicate the literature references at the end of this paper. at $\mathrm{A}$ and the flow splits so that, theoretically, equal amounts pass over the flow detectors, F. Column effluent enters at C, and also splits so that equal amounts flow to B and D, where the column effluent and reference gas mix and finally exit at $\mathrm{G}$. Thus, with the reference gas supplied to both the column and the detector, and no eluted compounds in the column effluent, a state of dynamic flow balance exists. Eluted compounds in the column effluent disturb the dynamic balance by causing an additional flow, $\Delta Q$, downward (if the eluate is more dense than the reference gas) in the center leg B-D. The disturbance in the dynamic equilibrium is transmitted into arms 1 and 2 , where its magnitude is measured by the transducers $\mathrm{F}$. One may note two fixed design characteristics at this point.

(1) Symmetry in the gas flows above and below the horizontal line ACG implies the requirement of symmetry in the duct dimensions about the same line.

(2) The driving force producing the disturbance in the flow rates is directly proportional [1] to the vertical height of the center section $\mathrm{B}-\mathrm{D}$ of figure 1 .

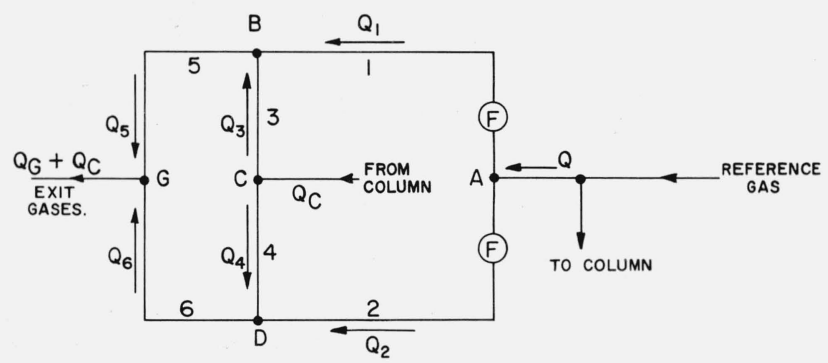

FIGURE 1. Diagrammatic representation of the Nerheim gas density balance showing location of detector units $F$, and directions of gas flow.

The lines represent ducts through which gases flow. 
Gravitational effects appear in the vertical sections of both branches 3-4 and 5-6 and cause changes in flow in each of the branches. However, if the flow transducers are to be kept out of contact with the column eluate in order to protect both the transducers and the sample, a third condition is imposed.

(3) Flow measuring elements are to be installed in branches 1 and 2 . This condition has certain implications with respect to reflection of flow disturbances into the ducts containing the transducers. It implies that, to be effective in protecting both transducers and sample, an operational factor must be controlled, namely, the flow in branches 1 and 2 should be high enough to prevent back diffusion of sample into these branches. As will be seen later, this condition results in a compromise in the selection of an appropriate flow rate.

At low Reynolds numbers (in this case 50), viscous flow occurs and Poiseuille's expression for the flow of a fluid through a conduit of circular cross section permits the construction of an electrical analog for the gas flows in the pneumatic system. To a first approximation the volume, $Q$, of gas flowing per unit time through a conduit of radius, $r$, and length, $L$, is given by

$$
Q=\frac{\pi P r^{4}}{8 \eta L}
$$

in which $P$ is the pressure-difference between the ends of the conduit and $\eta$ is the viscosity of the gas flowing through it. In the electrical analog the electrical current, I, corresponds to the "pneumatic current", $Q$; the electrical voltage drop, $E$, across a resistance corresponds to the pressure drop, $P$, between the ends of the conduit; and the electrical resistance, $R$, to the frictional resistance $\frac{8 \eta L}{\pi r^{4}}$ in the pneumatic system. The electrical analog for the pneumatic system in dynamic balance is shown in figure 2. The current measuring devices shown as $F_{1}$ and $F_{2}$ are presumed

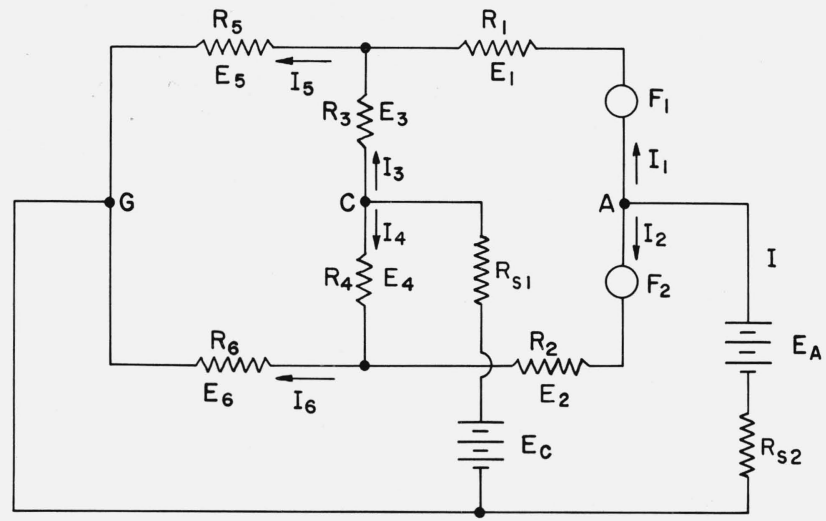

FigURE 2. Electrical analog for the pneumatic circuit of figure 1 showing current paths for the case when no sample is present in the column effluent, representing only reference gas flow through the balance.

$R_{s 2}$ is the source resistance for $E_{A}$. It consists of the regulator and needle valves. $R_{s 1}$ is the source resistance for $E_{c}$, consisting of an additional needle valve and the resistance of the column. Both $R_{s 1}$ and $R_{s 2}$ are high compared to the other resistances in the svstem. to be (in the case of the analog) the sensing elements of a differential ammeter. Condition 1 , requiring symmetry about the horizontal center-line stipulates that, for the electrical analog $R_{1}=R_{2}, R_{3}=R_{4}$ and $R_{5}=R_{6}$. Figure 2 represents the balanced pneumatic system in the absence of a sample in the column effluent, so that $I_{1}=I_{2}, I_{3}=I_{4}$ and $I_{5}=I_{6}$. The presence of an eluate, having a density greater than the carrier gas, causes a reduction in $Q_{3}$ and an increase in $Q_{4}$ (rates of flow in arms 3 and 4) of figure 1, which corresponds to a decrease in $I_{3}$ and an increase in $I_{4}$ of figure 2 . The total current in each part of the system may, therefore, be considered to be the sum of two currents - the one corresponding to the dynamic equilibrium in the absence of sample in the column effluent, and the other an internally circulating current due to the presence of such a sample. The differential flow detector is designed specifically to balance out the signals related to the flows associated with the dynamic equilibrium and to measure only the flow associated with the internal current caused by the difference in density of the sample. The electrical analog corresponding to the internal flow is shown in figure $3 \mathrm{a}$, and with lumped curcuit elements in figure $3 \mathrm{~b}$. The battery, $E$, in the figures, represents the pressure, $\Delta D g h$, causing the internal flow $(\Delta D=$ density difference between the contents of arms $\mathrm{A}$ and $\mathrm{C}$ of figure $1, g=$ the local acceleration of gravity, and $h=$ the vertical height of the gas column in the central arm, C, of fig. 1). The density difference also appears in the exit branch ( $\mathrm{G}$ of fig. 1) but its effect on the flows in the other branches will be neglected until after discussion of the circuit of figure $3 \mathrm{~b}$. For this circuit one may write

$$
I_{C}=I_{A}+I_{G}
$$

and

$$
E=I_{C} R_{C}+I_{A} R_{A}=I_{C} R_{C}+I_{G} R_{G}
$$

from which one obtains the expression

$$
E=I_{A}\left(R_{A}+R_{C}+\frac{R_{A} R_{C}}{R_{G}}\right)
$$

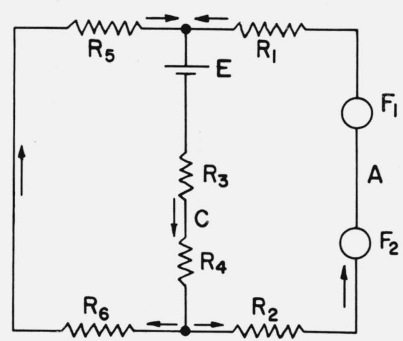

(a)

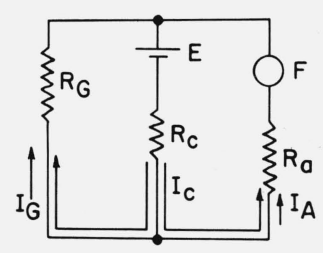

(b)
FiguRE 3. (a) Electrical analog of the pneumatic system of figure 1 showing only currents related to the presence of sample in the column effluent. (Internal gas flow). (b). The same as 3 a except that the circuit constants are lumped.

$R_{r}=R_{3}+R_{4}$ is the source resistance for $E$. It contains such factors as $L_{3}+L_{4}, \eta, r_{3}^{4}$ and $r_{4}^{4}$. 
Current flowing in the $\mathrm{G}$ branch is not measured by the detector, F, and thus, constitutes a loss in sensitivity. In order for the detector, $\mathrm{F}$, to measure a large fraction of the current, $I_{C}$, the resistance, $R_{G}$, must be large. If $R_{C}+R_{A} \gg \frac{R_{A} R_{C}}{R_{G}}$ the last term in eq (4) may be neglected, and $I_{A}=I_{C}=I$. The sensitivity, in terms of units of flow per unit of pressure becomes

$$
\frac{I}{E}=\frac{1}{R_{C}+R_{A}}=\frac{1}{4 R}
$$

in the electrical analog. The above considerations lead to the design condition:

(4) The pneumatic resistance in the $G$ branch should be high compared to that in the $\mathrm{A}$ and $\mathrm{C}$ arms of figure 1 . Considering symmetry requirements along with condition 4 , leads to

$\frac{L_{5}}{r_{5}^{4}}+\frac{L_{6}}{r_{6}^{4}} \gg \frac{L_{1}}{r_{1}^{4}}+\frac{L_{2}}{r_{2}^{4}}+\frac{L_{3}}{r_{3}^{4}}+\frac{L_{4}}{r_{4}^{4}}$ and $\frac{L_{5}}{r_{5}}=\frac{L_{6}}{r_{6}}, \frac{L_{1}}{r_{1}}=\frac{L_{2}}{r_{2}}, \frac{L_{3}}{r_{3}}=\frac{L_{4}}{r_{4}}$.

For that part of the circuit involving only the A and $\mathrm{C}$ branches of figure $3 \mathrm{a}$, the total resistance around the circuit is $R_{1}+R_{2}+R_{3}+R_{4}$. The pneumatic equivalent of the Ohms law equation becomes:

$$
\Delta Q=\frac{\Delta D g h}{\frac{8 \eta^{*}}{\pi}\left(\frac{L_{1}}{r_{1}^{4}}+\frac{L_{2}}{r_{2}^{4}}+\frac{L_{3}}{r_{3}^{4}}+\frac{L_{4}}{r_{4}^{4}}\right)} .
$$

If $L_{1}+L_{2}+L_{3}+L_{4}=L$ and $r_{1}=r_{2}=r_{3}=r_{4}=r$

$$
\Delta Q=\frac{\pi g h r^{4}}{8 \eta L} \Delta D .
$$

For convenience, the assumptions used in the derivation of eq (6) are summarized and discussed below.

1. It was assumed that the A- and C-branches had the same (low) resistance to flow and that the G-branch had high resistance to flow. Since most potentiometric recorders cannot resolve signals much smaller than about 0.5 percent there is little point in making the total resistance in the $\mathrm{G}$-branch more than 200 times that in the A- and C-branches. Note that, in the derivation of eq (6), it cannot be assumed that $R_{g}=\infty$ because this assumption would lead to a system with two inlets and no outlet, and the balance could not work. However, a moderate increase in the overall pressure drop could increase the sensitivity by way of an increase in $\Delta D$.

2. The assumption of laminar flow in the A- and Cbranches appears to be justifiable for Reynolds numbers of about 50 and below.

3 . The viscosity of the carrier gas was considered to be identical to that of the reference gas. For the case of ducts of uniform radius, the resistance to flow around the $\mathrm{A}$ - and $\mathrm{C}$-branch loop is proportional to $\eta_{1}\left(\frac{L}{2 r^{4}}\right)+\eta_{2}\left(\frac{L}{2 r^{4}}\right)=\frac{\eta_{1}+\eta_{2}}{2}\left(\frac{L}{r^{4}}\right)$. Depending somewhat on the column and the nature of the sample gas, the actual concentration of sample in the carrier gas is of the order of 0.5 percent. If it is assumed that the viscosity change on mixing is proportional to the amount of sample in the carrier gas, the change in resistance to flow would have a maximum value of 0.5 percent of the average of their viscosities. Additionally, the small error from this source would become smaller with an increase in detector sensitivity which would permit the use of a smaller sample.

4. The effects of gravitationally induced flow in the G-branch on the internal flow measured in the A-branch would be small for two reasons. Such a flow must overcome a resistance which is large compared to that in the A- and C-branches taken as a parallel circuit and secondly, the force producing the flow in the G-branch is less than that in the C-branch by a factor of $\frac{Q_{c}}{Q_{c}+Q}$ because of dilution at points $\mathrm{B}$ and $\mathrm{D}$ of figure 1 . For example, if $R_{(i}=100\left(R_{C}+R_{A}\right)$ and for $Q_{C}=25$ and and $Q=150 \mathrm{~cm}^{3} \mathrm{~min}^{-1}$ the amount of flow reflected into the A- and C-branches would be only 0.14 percent of the internal flow produced by $\Delta D g h$ in the C-branch.

5. The flow measuring devices ( $F$ in fig. 1 ) were assumed to introduce no resistance to flow in the A-branch. The error introduced by this assumption depends on the skill of the designer, but presumably it can be made small at the low velocities expected.

6. The effects of T-joints and $90^{\circ}$ bends on the resistance to flow in the A- and C-branches were assumed to be negligible. Such resistances depend primarily on the velocity of gas flow, and at constant flow rate, they are also constant. Some estimates of the magnitude of such resistances in relation to the resistance of the straight sections of the ducting are made in the appendix, q.v. For a balance having ducting of $1 / 4$ in diam with a reference gas $N_{2}$ flow rate of $150 \mathrm{~cm}^{3} \mathrm{~min}^{-1}$, and a carrier gas flow rate of $25 \mathrm{~cm}^{3} \mathrm{~min}^{-1}$, a height of 45 to $60 \mathrm{~cm}$ would be required to reduce the error caused by the neglect of resistances in T-joints and bends to 1 percent of that in the straight sections, the required height being a function of the ratio of the radius of curvature of the elbows to their pipe radius.

In accordance with the notation of figure 1 , let $Q_{0}$ be the flow in each of the branches 1 and 2 when no sample is present in the central branch. When sample is present $Q_{1}=Q_{0}+\Delta Q$ and $Q_{2}=Q_{0}-\Delta Q$ in which $\Delta Q$ is the volume rate of internal flow associated with the density difference $\Delta D$. The detectors are sensitive to velocity rather than to volumetric flow rate. Thus, for ducts of equal diameter one may write:

$$
\frac{Q_{1}-Q_{2}}{Q_{0}}=\frac{2 \Delta Q}{Q_{0}}=\left(\frac{\pi g h r^{4}}{8 \eta L Q_{0}} \Delta D\right) \times 2
$$


Since velocity is directly related to volumetric flow rate

$$
\frac{v_{1}-v_{2}}{v_{0}}=\frac{2 \Delta_{v}}{v_{0}}=\frac{\pi g h r^{4}}{2 \eta L Q} \Delta D
$$

in which $Q=2 Q_{0}$ is the total amount of reference gas flowing per second into the system at point $A$, figure $1, \Delta v$ is the velocity of internal flow and $v_{0}$ is the velocity in branches 1 or 2 when no sample is present. Equation 7 indicates that, subject to the foregoing design limitations and at constant operating conditions, the relative change in velocity is linear with respect to the density difference between the contents of branches $\mathrm{A}$ and $\mathrm{C}$.

Equation (7) contains no references to values applicable to the exit branch, $G$, it being required only that the resistance in this branch be sufficiently large. The requirement for high resistance is reduced somewhat when the effect of gravitation in the exit branch is considered. Figure 4 is a modification of figure $3 b$ to show the effect of gravitational pressure in the exit branch. $E_{G}$ is polarized in such a direction as to oppose the flow of internal current in the G-branch and adds to the voltage drop across $R_{G}$ produced by the internal current.

\section{Measurement of Gas Velocities}

Hot wire anemometers are conventionally used as transducers for the conversion of gas velocities into electrical signals. One of the requirements imposed on the transducer is that it liberate little heat energy into the gas stream. Such heat release can result in stack action (chimney effect) producing an internal flow independent of the density difference which is to be measured. On the other hand, the transfer of heat energy to the gas stream from the hot anemometer element is the principle on which conventional anemometers operate. Obviously a compromise is in order, and a limit is set on the ultimate sensitivity attainable, which depends, somewhat, on the location of the anemometers in the pneumatic system. While thermistor elements have higher temperature coefficients of resistance and may liberate somewhat less heat for a comparable sensitivity than do wire elements, they are subject to drift and are hard to obtain in pairs sufficiently well matched both thermally and electrically.

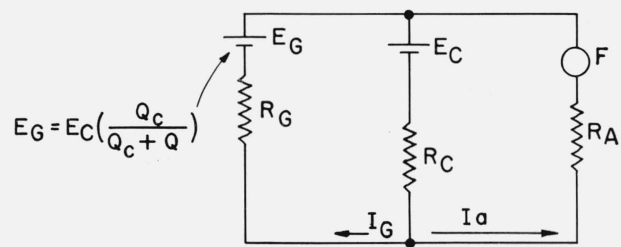

FIGURE 4. Electrical analogue circuit of figure $3 b$ with potential source added to represent gravitational forces in the exit branch. (Internal gas flow only) $R_{g}$ is the source resistance of $E_{f i}$. It includes such factors as $L_{5}+L_{6}, \eta, r_{5}^{4}$ and $r_{6}^{4}$.
Their use has been covered elsewhere [1]. Wire elements must be made from small enough wire (in the order of $0.0001 \mathrm{in}$ ) to be sufficiently sensitive and liberate negligible amounts of heat energy. Their disadvantage is that they are fragile. When properly aged they are subject to much less drift than thermistors, and their time constants are negligible compared to that of the usual strip-chart recorder. In general, the properties of hot wire anemometers appear to be adequate for the intended use, particularly if the design variables of both the electrical and pneumatic systems are optimized.

Hot wire anemometers may be used either as constant resistance or as constant current devices [5]. Operation as constant resistance devices implies certain difficulties in that a single anemometer must be used and the voltage to the Wheatstone's bridge or the current through it must be varied in such a way as to maintain bridge balance. Conversely, operation of a pair of anemometers as constant current devices presents few problems and has the added advantage that a properly connected bridge can be self-compensating with respect to current changes caused by resistive changes in the sensing elements. Since the resistive change in one anemometer is equal and opposite to that in the other, the total bridge resistance remains constant, as does the voltage across the bridge. Such a bridge is shown in figure 5. It should be noted that the circuit of figure 5 is not a true Wheatstone's bridge in that the potentiometric recorder bucks out all voltage across the "galvanometer arm" of the bridge so that no current flows through it. The circuit should be regarded as a fixed and a variable voltage divider connected in parallel, the junction between resistors $S_{1}$ and $S_{2}$ acting as the fixed reference potential.

The velocity, $v$, of gas flow over a hot wire anemometer element is related to the resistance of the element at operating temperature, $R$, and its resistance at ambient temperature, $R_{a}$, by the equation

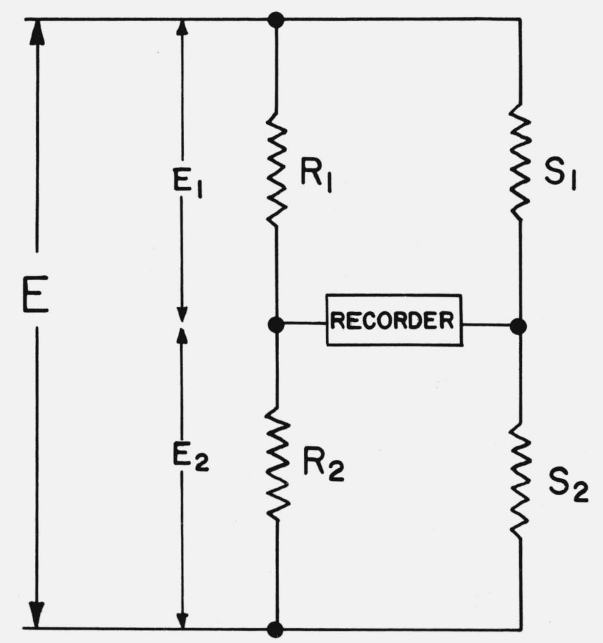

FIGURE 5. The electrical measuring circuit showing the connec tions to the anemometers, $\mathrm{R}_{1}$ and $\mathrm{R}_{2}$, and to standard resistors $\mathrm{S}_{1}$ and $\mathrm{S}_{2}$. 


$$
\frac{I^{2} R}{R-R_{a}}=M \sqrt{v}+B
$$

in which $I$ is the current supplied to the element. If $I$ is made constant, it may be combined with $M$ and $B$ to give

$$
\frac{R}{R-R_{a}}=m \sqrt{v}+b
$$

The straight-line relationship of figure 6 applies only at velocities sufficiently high so that the effects of thermal convection are negligible. Below this particular velocity the slope falls off and approaches zero or even reverses in sign [5]. A typical curve, obtained by calibration in the instrument, is shown in figure 6.

Proper matching of a pair of anemometers implies another design criterion.

(5) Within "reasonable" limits, the values of $m$ and $b$ in eq (13) should be the same for each of a pair of matched wire anemometers.

If $m_{1}$ is assumed equal to $m_{2}$ and $b_{1}=b_{2}$ for such a matched pair, the fractional change in resistances must be equal, i.e., $\frac{R_{1}-R a_{1}}{R_{1}}=\frac{R_{2}-R a_{2}}{R_{2}}$, for the same velocity of gas flow across them. In order for them to show this characteristic it is impractical that they be physically very different so that it is reasonable to assume that $R a_{1}=R a_{2}=R a$, and, for equal velocities of flow across the wires (no sample in the C-branch), $R_{1}=R_{2}=R$. For the off-balance condition, with internal flow present, $R_{1}=R-\Delta R$ and $R_{2}=R+\Delta R$, assuming the sample to be more dense than the carrier gas. The

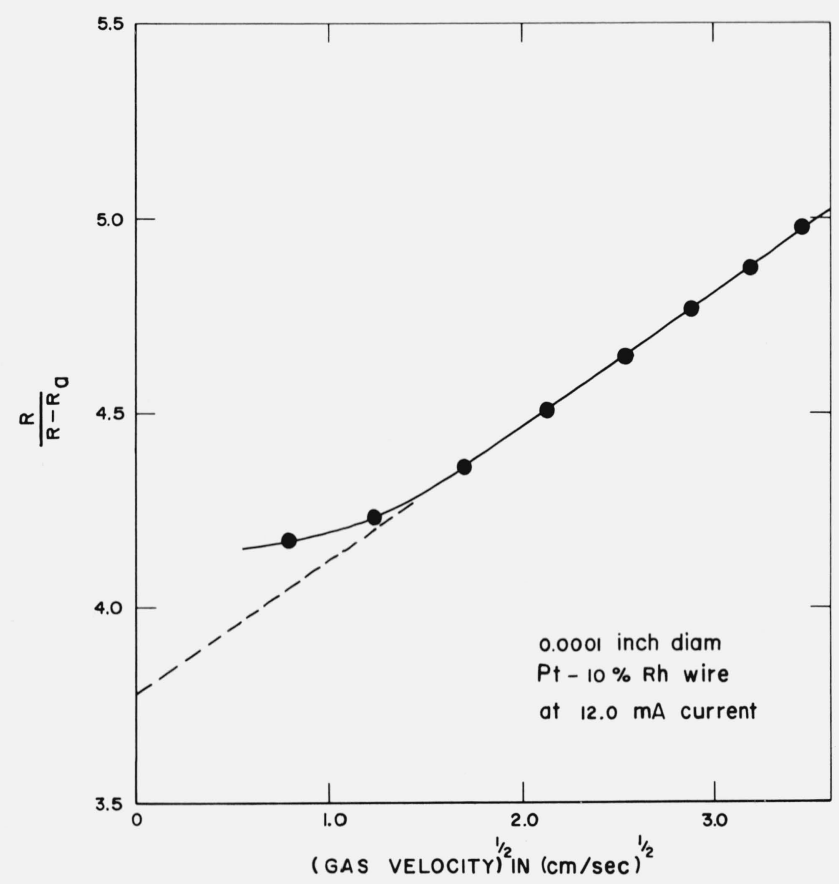

Figure 6. Calibration curve for a hot wire anemometer, showing the effect of convection currents at low gas velocities. fractional change in the velocity caused by the internal flow produces resistive changes given by

$$
\begin{aligned}
& \frac{v_{1}-v_{2}}{v_{0}}=\frac{2 \Delta v}{v_{0}} \\
& =\frac{\left[\frac{R-\Delta R}{R-\Delta R-R a}-b\right]^{2}-\left[\frac{R+\Delta R}{R+\Delta R-R a}-b\right]^{2}}{\left[\frac{R}{R-R a}-b\right]^{2}}
\end{aligned}
$$

which simplifies to

$$
\frac{\Delta v}{v_{0}}=2 \Delta R\left[\frac{-R a}{R(R-R a)-b(R-R a)^{2}}\right]=2 K \Delta R
$$

in which .

$$
K=\frac{-R a}{R(R-R a)-b(R-R a)^{2}} .
$$

The negative sign is an indication that the slope of the $\frac{R}{R-R_{a}}$ versus $v^{\frac{1}{2}}$ line is opposite to that of the $R$ versus $v^{\frac{1}{2}}$ line. The sign of $K$ has no practical significance since it is easily taken care of by reversing the polarity of the batteries driving the bridge. Substituting from eq (7)

$$
\Delta R=\frac{1}{K}\left(\frac{\pi g h}{8 \eta L}\right) \frac{r^{4}}{Q} \Delta D
$$

The signal to the recorder, $\Delta E$, is $I \Delta R$, considering the wires in series as a simple voltage divider, so that

$$
\Delta E=\frac{\pi g h r^{4} I}{8 \eta K L Q} \Delta D .
$$

If the current, $I$, is expressed in milliamperes, the overall sensitivity in units of millivolts per gram $-\mathrm{cm}^{3}$ is

$$
\frac{\Delta E}{\Delta D}=\frac{\pi g h r^{4} I}{8 \eta K L Q}
$$

\section{Detector Volume}

The volume of sample plus carrier gas included in the detector is of major concern where either the size of the available sample is small or where minor constituents of a mixture are to be determined. The rather large dividends in detector sensitivity realized from rather small increases in its radius requires that some consideration be given to the effects of the increased detector volume on the shapes of recorded peaks. When the area under the peak is to be used as a measure of the amount of an eluted constituent, it is imperative that peaks do not overlap, if the area 
measurements are to be made accurately. The separation of recorded peaks depends on both the detector and the column and, to a certain extent, the overlapping caused by a large detector volume can be compensated for by a redesign of the column. It seemed desirable, therefore, to estimate the increase in the sensitivity of the detector in terms of the increase in peak width resulting from an increase in detector volume, assuming the increase in volume to be obtained by way of an increased radius, the height remaining fixed. It should be noted that the volume of interest is that of the C-branch alone.

A qualitative understanding of the effects of detector volume in a real detector operating on a real sample can be had by comparison with a hypothetical detector working on a hypothetical sample. Assume a hypothetical sample of one arbitrary unit of volume, contained in a tube between two sections of carrier gas, from which it is discharged into a hypothetical detector, the volume of which may be changed at will without affecting its sensitivity. It will further be assumed that no mixing occurs so that the sample has planar leading and trailing edges and that it is introduced directly (no column) into the detector. It may be assumed, first, that the detector has infinitely small volume so that it is either completely filled or completely empty of sample at any given instant. The output of such a detector with such a sample would be a square wave. A detector having the same volume as the sample is more realistic. Its output would take the form of an isosceles triangle, the detector being completely filled at only one instant. Similarly, for detectors having volumes greater than the hypothetical sample, the out put would have the shape of an isosceles trapezoid. In the above examples, three effects are to be noted. (1) When the detector volume exceeds one unit (equal the sample size) the maximum response decreases. (2) The width of the curve increases with increase in detector volume. (3) The area under the curve remains constant, because the detector measures all of the sample that passes through it to give a quantitative measure of the total amount of sample. For the case where some mixing can occur, the sharp corners of the curves would be rounded, the exit corners being somewhat more rounded than the inlet corners because of the increased time allowed for mixing.

Consider, next, the effect of the column on the same hypothetical sample. The effect is to retain the individual molecules for different times, depending on their individual energies, so that, at the column exit, the concentration would vary to approximate a normal distribution curve [6]. The hypothetical "ideal" detector, having zero volume, would follow the instantaneous concentration perfectly and give a recorded output which would also be a normal distribution curve. However, a nonideal detector, having a finite volume and responding to a constantly changing sample concentration, would give an instantaneous output corresponding to the average density of the contents of its C-branch. Its recorded peak would be a modified normal distribution curve, being somewhat wider and not as high at the maximum as that from the detector having zero volume. Thus, the larger the volume of the detector, the greater the area of the normal distribution curve which would be instantaneously averaged, and the lower and broader the recorded output would become.

The practical gas density balance more than compensates for the theoretical reduction in curve height, however, since its sensitivity increases as the square of its volume at constant detector height. The extent of the overcompensation is shown in figure 7 for some of the cases discussed above. Since a practical detector would have zero sensitivity at zero volume, no curves are shown for these cases. Curve A represents the output for a practical detector of one arbitrary unit of volume responding to a hypothetical sample of one unit of volume when the sample is introduced directly into the detector. The output would increase linearly until the detector was completely filled, then decrease linearly until it was completely empty. Curve B represents a similar situation except that the volume of the detector was taken as two arbitrary units. When the sample has been completely transferred to the detector, the sample represents only 50 percent of the total contents. However, the maximum output would be $0.5 \times(2)^{2}=2$. Curve $C$ represents the output of a detector of three arbitrary units of volume when supplied with a sample of an arbitrary unit of volume. Similar reasoning was applied to the case where the sample was assumed to have passed through a column. For the output of the detector having zero volume, the coordinates were chosen such that the area under the curve was equal to one arbitrary unit. This curve was scanned by assumed detector volumes of $1,2,3$, and 4 units and output values calculated. The curves were cut off at 99.9 percent of the full area under the curve and matched on the left to simulate the appearance of a sample at the detector inlet. They are shown as curves D, E, F, and $\mathrm{G}$ of figure 7. The areas under these four curves were plotted in figure 8 as a function of the width. Figure 8 indicates that an increase in detector volume of four units produced an increase in area of 1340 percent at a cost of about 30 percent in increased width.

\section{Choice of Reference Gas}

Equation 18 may be rewritten as $\Delta E \propto \frac{\Delta D}{\eta}$ assuming all the operational and design parameters to be constant and assuming the carrier gas to be the same as the reference gas. For a fixed volumetric concentration of sample in the carrier gas one may write $\Delta E \propto \frac{D_{s}-D_{r}}{\eta}$ in which $D_{r}$ is the density of the reference gas and $D_{s}$ is the density of the sample. If one further assumes that the sample has a density of $1 \mathrm{~g} / \mathrm{cm}^{3}$, one may calculate a relative response to be expected from several reference gases. This has been done and is shown in table 1 . The gases in the list were chosen because 


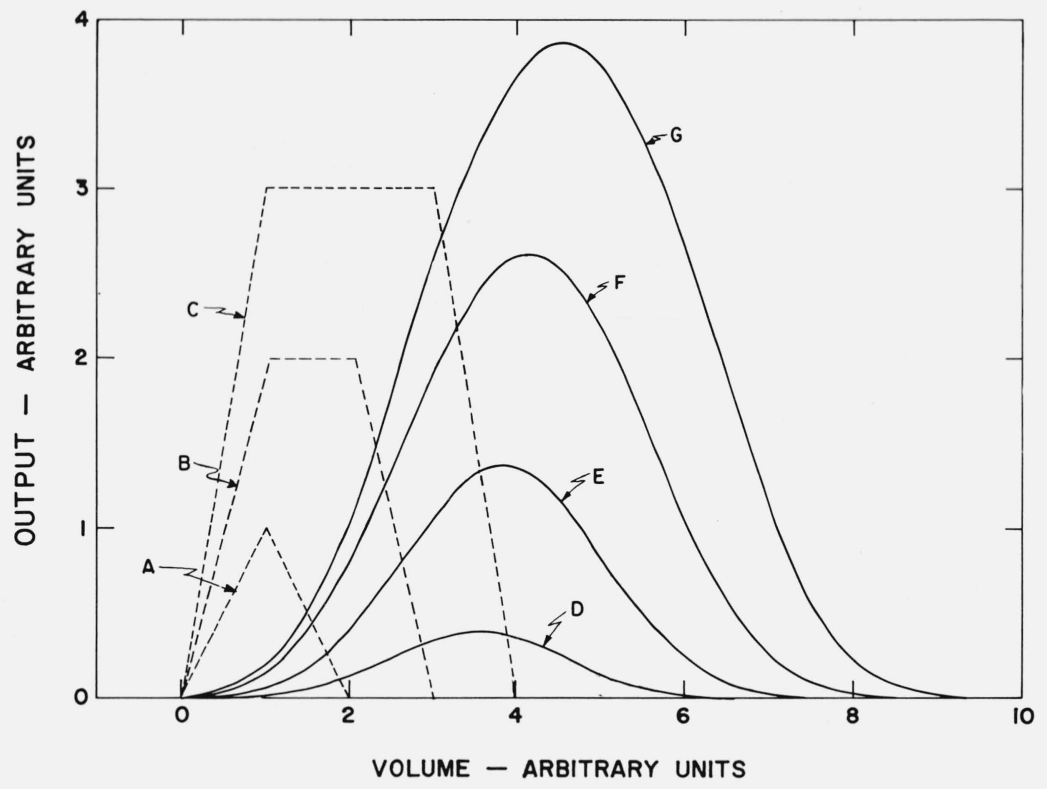

FIGURE 7. The effect of increased detector output associated with increased detector volume at constant height.

Curves A, B and C are for a hypothetical slug of sample having planar ends traversing detectors of volumes: A. equal to the sample volume; B- twice the sample volume, and C-three times the sample volume. Curves D, E, F and $\mathrm{G}$ show the effect on detector output of the same l-unit-of-volume sample after passing through a column, for detectors having volumes $1,2,3$, and 4 times that of the sample.

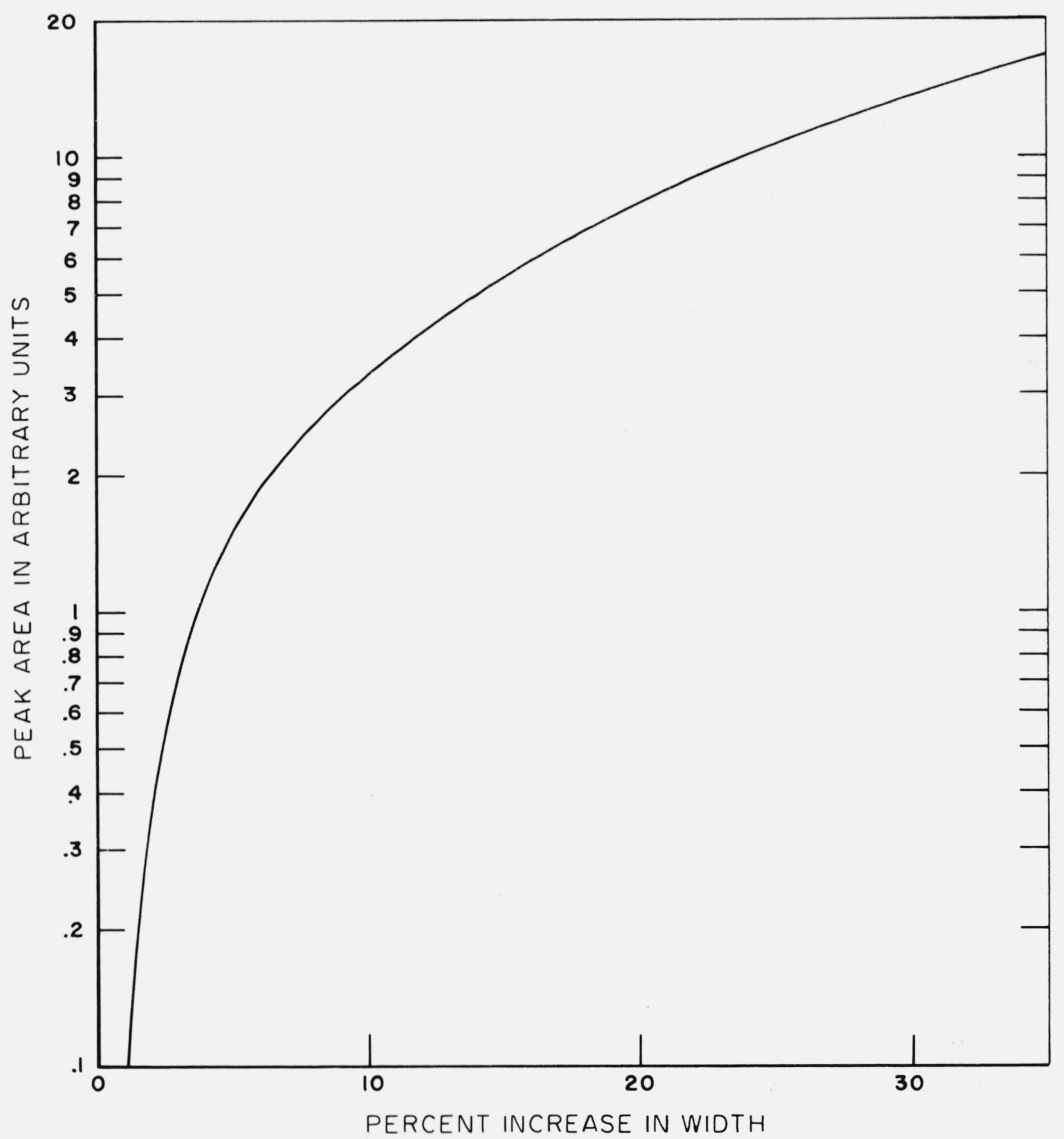

FIGURE 8. The increase in peak area obtained at the expense of peak width when the detector volume is increased, the height remaining constant. 
TABLE 1

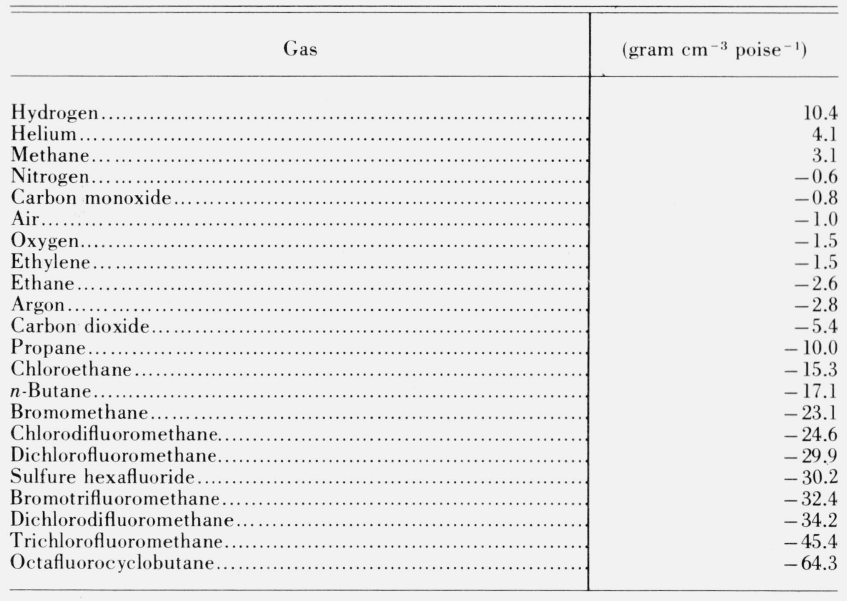

information on both density and viscosity were available. They were listed without regard to their toxic hazards or their suitability for use as chromatographic carrier gases. Such a table is useful in that the relative response for one of the gases as the sample in another as the reference gas may be obtained from the table.

Guilleman and Auricourt [7] made measurements of the effects of reference gases of different density on the performance of the gas density balance. They neglected the effects of flow rate, $Q$ in eq (18), on sensitivity and the effects of using different carrier gases on resolution of the column. Accordingly, their results cannot be compared with those predicted from the table.

\section{Discussion}

An order of magnitude value for the probable sensitivity of the detector is of interest. Equation 18 may be rewritten as:

$$
\frac{\Delta E}{\Delta D}=2.18 \times 10^{6}\left(\frac{h}{L}\right)\left(\frac{I}{K}\right)\left(\frac{r^{4}}{Q}\right) \text { millivolt per gram-cm }{ }^{-3}
$$

assuming room temperature and nitrogen as the reference gas. Values of the three quantities in parentheses will be estimated separately starting with the last one.

The results shown in figure 6 were obtained on an anemometer wire mounted horizontally in a $0.635 \mathrm{~cm}$ (1/4 in) I.D. hole in a brass block. The value of $r$ was, therefore, fixed at $0.3175 \mathrm{~cm}$ and the smallest usable value of $Q$ can be estimated from the curve of figure 6 . However, the anemometers operate in opposite directions on the curve, and an operating point must be chosen which will allow each anemometer to operate in the linear region of its curve. For purposes of estimation we may choose to use the point at $\sqrt{v}=2$. The value calculated for $Q\left(Q=2 Q_{0}\right)$ was $2.53 \mathrm{~cm}^{3} / \mathrm{sec}$ $\left(152 \mathrm{~cm}^{3} \mathrm{~min}^{-1}\right)$ giving a value for $\frac{r^{4}}{Q}=4.01 \times 10^{-3} \mathrm{~cm}$

Values for $\frac{I}{K}$ have been calculated from directly measured anemometer characteristics at measured currents for the anemometer of figure 6 . At $12 \mathrm{~mA}$ current, $\frac{I}{K}$ has a value of $130 \mathrm{mV}$. ( $K$ has the dimensions of reciprocal ohms so that $\frac{I}{K}$ has the dimensions of milliampere ohms or millivolts.)

Assuming that $L=2 h$ and combining all of the above estimates gives an overall estimated detector-sensitivity of $0.56 \times 10^{6} \mathrm{mV} / \mathrm{g}-\mathrm{cm}^{-3}$.

At a bridge current of $25 \mathrm{~mA}$, a little over $50 \mathrm{~mW}$ is dissipated into and carried away by the reference gas. When the sample is absent, the heat is carried equally into each of the arms of the A branch, and, because of the vertical sections in the A branch, a certain amount of chimney effect is produced. When sample is present, the internal gas flow adds algebraically to the reference gas flow and the distribution of heat energy is changed, causing a change in the amount of chimney effect. The effect on the record of an eluted peak would be a failure to return promptly to zero, or to return to a displaced but reasonably constant zero because the effect of the changed heat distribution persists for some time after the sample has passed. For this reason, the amount of energy dissipated into the gas stream should be kept small, the reference gas ducts should be kept close together for some distance on both sides of the anemometers, and the mounting block should be made of a metal having a high thermal conductivity to minimize temperature differences.

From the above, it appears that the Nerheim gas density balance is affected by several design variables which, if properly chosen, can make it suitable for a number of applications involving either high or low sensitivity and large or small sample sizes. It is also subject to limitations which should be considered in its applications.

\section{Appendix}

Head loss coefficients for T-joints and $90^{\circ}$ bends have been measured by a number of authors $[8,9,10]$ at relatively high Reynolds numbers but actual measurements at low Reynolds numbers are scarce and none have been discovered which seem to apply to the conditions encountered in the gas density balance. Various schemes of extrapolation of the data into the laminar region have been proposed. Perhaps that of Prandtl [11] for use with $90^{\circ}$ bends is the best known. The coefficient of resistance of the curved pipe, $\lambda$, is related to that of a straight pipe of the same length, $\lambda_{0}$, by the equation

$$
\frac{\lambda}{\lambda_{0}}=0.37 D^{0.36}
$$

in which $D$ is the Dean number, $\frac{R e}{2}\left(\frac{\mathrm{r}}{r_{c}}\right)^{1 / 2}$, with $R e$ the Reynolds number and $r_{c}$ and $r$, the radius of curvature and the pipe radius, respectively. This equation is reported [12] to apply between $10^{1.6}<\operatorname{Re}\left(\frac{r}{r_{c}}\right)^{1 / 2}<10^{3}$. However, at low Reynolds numbers and low values of 
$\frac{r}{r_{c}}$ the ratio $\frac{\lambda}{\lambda_{0}}$ does not extrapolate to 1.0 as might be expected: e.g., at $\operatorname{Re}=50$ and $\frac{r}{r_{c}}=100, \frac{\lambda}{\lambda_{0}}=0.51$. Walker, Lewis, McAdams, and Gilliland [13] give an empirical equation for estimating the loss coefficient $K$, for Reynolds numbers between 100 and 1000 and for $R e<10$, which does not include the range of interest here. However, using the data of Walker, et al., there has been developed [9] an expression which gives a practical estimate of losses in ordinary pipe fittings, T's and $90^{\circ}$ elbows. Resistances to flow calculated for pipe fittings are probably somewhat greater than for similar joints in glass tubing, but will serve to give better than order-of-magnitude estimates on which to base the neglect or inclusion of such resistances in the A- and C-branch loop of figure 1 .

The loss of head, $h$, in such pipe fittings as above is given in terms of the square of the velocity, $V$, the local acceleration of gravity, $g$, the friction factor, $f$, and the ratio of the length to diameter of the pipe, $\frac{L}{2 r}$.

$$
h=f\left(\frac{L}{2 r}\right) \frac{V^{2}}{2 g}
$$

In the case of pipe T's and elbows it is customary to give a value of $\frac{L}{2 r}$ which is larger, by some experimentally determined factor, than that for a straight section so that the head loss of the fitting is expressed as equivalent to a length of straight pipe in terms of its diameter. For laminar flow, the equivalent length,

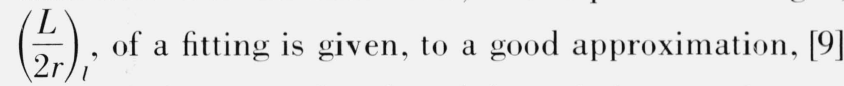
in terms of its equivalent length for turbulent conditions, $\left(\frac{L}{2 r}\right)_{t}$, by $\left(\frac{L}{2 r}\right)_{1}=\frac{R e}{1000}\left(\frac{L}{2 r}\right)_{t}$. The head loss for laminar flow, $h_{l}$, then becomes $h_{l}=f_{l} \frac{R e}{1000}\left(\frac{L}{2 r}\right)_{t} \frac{V^{2}}{2 g}$. The friction factor, $f_{l}$, for laminar flow is $\frac{64}{R e}$ so that $h_{l}=0.064\left(\frac{L}{2 r}\right), \frac{V^{2}}{2 g}$ or $h_{l}=K \frac{V^{2}}{2 g}$. Values for $\left(\frac{L}{2 r}\right)_{t}$ from reference $[9]$ indicate that the bend resistance goes through a minimum at $r_{c} \cong 3.7 r$, and approaches a maximum value of about 18 at $r_{c} \geqq 36 r$. For flow through the run of a $\mathrm{T},\left(\frac{L}{2 r}\right) \cong 20$ and when the flow is through the branch $\left(\frac{L}{2 r}\right)_{t} \cong 60$. The corresponding resistance coefficients are:

For $90^{\circ}$ bends

For $90^{\circ}$ bends

For $T$, through run

For T, through branch
$K_{B}=0.24$ minimum

$K_{B}=1.15$ maximum

$K_{r}=1.28$

$K_{b}=3.84$.
The head loss may be converted into resistance to flow, $\frac{\Delta P}{Q}$, by substituting $V=\frac{Q}{\pi r^{2}}$ and $\Delta P=\rho g h$ into the above so that resistance to flow through a fitting $=\frac{K}{2 \pi^{2} r^{4}} \rho Q$. Summing the resistances for the bends and T's in the A- and C- branches and dividing by the total resistance, including the straight sections, the fraction of the total resistance due to the bends and T-joints becomes (assuming $\rho_{1}=\rho_{2}$ )

$$
\frac{\rho\left[K_{r}\left(\frac{3 Q+Q_{c}}{2}\right)+K_{b} Q_{c}+K_{B} Q\right]}{16 \pi \eta L+\rho\left[K_{r}\left(\frac{3 Q+Q_{c}}{2}\right)+K_{b} Q_{c}+K_{B} Q\right]}
$$

Reasonable values of the quantities in the above expression are as follows:

$\rho=1.1457 \times 10^{-3} \mathrm{~g}-\mathrm{cm}^{-3}\left(N_{2}\right.$ at room temp. and $\left.1 \mathrm{~atm}.\right)$

$Q=150 \mathrm{~cm}^{3} \mathrm{~min}^{-1}=2.5 \mathrm{~cm}^{3} \mathrm{sec}^{-1}$

$Q_{c}=25 \mathrm{~cm}^{3} \mathrm{~min}^{-1}=0.417 \mathrm{~cm}^{3} \mathrm{sec}^{-1}$

$\eta=0.181 \times 10^{-3}$ poises $\left(N_{2}\right.$ at room temp. and $1 \mathrm{~atm}$. $)$

Substituting these values into the above and solving for the percentage of the total resistance which occurs in the T-joints and bends gives $L=\frac{120}{E}-1.2$ for the case where $r_{c}=36 r$ and $L=\frac{91.5}{E}-0.92$ for $r_{c}=8 r$, in which $E$ is the error, in percent, caused by the neglect of bends and T-joints.

\section{References}

[1] Nerheim, A. G., Anal. Chem. 35, 1640 (1963).

[2] Martin, A. J. P., U. S. Patent 2,728,219 (Dec. 27, 1955).

[3] Martin, A. J. P., and James, A. T., Biochem J., 63, 138 (1956).

[4] Able, K., and Noble, F. W., Trans. N.Y. Acad. of Sci. Ser. II, 26 (No. 2) 159 (1963).

[5] Ower, E., The Measurement of Air Flow (Chapman and Hall Ltd., London, 1949).

[6] Haarhoff, P. C., and Vander Linde, H. J., Anal. Chem. 28, 573-82 (1966).

[7] Guillemin, C. L., and Auricourt, A., J., Gas Chromatography 2, 156-159 (1964).

[8] Beij, K. H., J. Res. NBS 21, 1-18 (1938) RP1110.

[9] Flow of Fluids Through Valves Fittings and Pipe, Tech. Paper No. 410 (1965), Crane Co., Chicago.

[10] Ito, H., ASME Trans., Series D., J. Basic Eng. 82, 131-143 (1960).

[11] Prandtl, L., Führer durch die Strömungslehre, 3d ed. p. 159, Braunschweig (1949).

[12] Schlichting, H., Boundarv Layer Theory, p. 530 (McGraw-Hill Book Co., Inc., New York, N.Y., 1960).

[13] Walker, W. H., Lewis, W. K., McAdam, W. H., and Gilliland, E. R., Principles of Chemical Engineering (McGraw-Hill Book Co., Inc., New York, N.Y., 1937). 